\title{
The National Schizophrenia Fellowship on treatment guidelines ${ }^{\dagger}$
}

The National Schizophrenia Fellowship (NSF) believes that people should be offered choice and the means to exercise that choice.

We are the largest mental health charity in Europe that works with, and on behalf of, people living with a severe mental illness (SMI) and their carers. Developments in treatment have understandably been closely monitored since the charity's origins in the early 1970s. Our information and advice comes from a wide range of sources, but most importantly from the 10s of 1000s of people living with a SMI who are in contact with the NSF each year.

The NSF believes in a holistic approach to mental illness, where someone's need for accommodation, occupation, money, social life and physical as well as mental health care all contribute to a positive mental health outcome. It is our experience that the new medicines are on the whole preferred by the people who we work with and who we represent. We have therefore been campaigning against the rationing of these medicines, which has been blatant in some areas of the UK. The NSF has produced two reports on the subject (Is cost a factor? Hogman, 1996 and Is cost a Factor - II? Taylor et al, 1999). The NSF has also been involved in national media campaigns to raise awareness of this scandal.

The advantage of the new medicines is their different side-effect profile, which has increased the range of choice. The apparent reduction in extra pyramidal side-effects, the 'numbing' and 'knock-out' sideeffects especially, has greatly increased the quality of life of people who experienced them. The effect on negative symptoms is also cited by many, especially families who see their relatives' personalities return.

However, not everyone has found the atypicals beneficial. A number have returned to the older medicine, often citing weight gain as the motivating factor. There is no single 'best' medicine for people with schizophrenia. However, for those with refractory experience there is much evidence that clozapine is effective in up to twothirds of cases. Arguably some medicines appear to have more going for them than others and where experienced doctors talk about these it is the side-effect profile, not the effectiveness on positive symptoms, that makes the difference. As the various side-effects are viewed with differing importance it is necessary to take time to consult and involve individuals, doctors and carers before identifying a 'best fit' option that enhances people's quality of life.

To find their 'best fit' people require good information, including the risks involved, and a choice of interventions. For this to be achieved, as it already has been in some UK services, there should be no rationing of safetyapproved medicines or therapies. Rationing is usually linked to financial cost but in this context also relates to limitations imposed by a lack of personnel, skills or poor procedures. When you consider the painful, disabling and life threatening effects of an illness like schizophrenia, the additional cost of the newer drugs, at around $f 2000$ per person per year - even assuming that no savings in bed usage or other services take place - is small in relation to the increase in people's quality of life.

Clinicians and medical staff need to receive comprehensive training in new treatments, as they become available. They also need to meaningfully involve individuals in making decisions about treatments. This includes giving them clear information about what the medicine is, how it works, what side-effects are associated with it (and what can be done to alleviate these) and when it might have an effect. A written plan should also be supplied and information about review of the medicine rights to a second opinion and advocacy should be freely available. People with mental illness, and also their families, have responsibilities within the partnership to stick to a care plan once it has been agreed and fully inform the care team of changes in their experience.

A number of these issues are being debated at the moment in connection with the Mental Health Act Green Paper, the work of the National Institute for Clinical Excellence (NICE) and the National Service Framework. One of the 'milestones' in the framework for mental health states: "Using clinical guidelines, all service users should be assessed for and receive new antipsychotics where indicated" (NHS Executive, 1999).

The clinical guidelines referred to in the above statement will be supplied by NICE, probably at some point in 2001. Its guideline development process will review all current evidence around treatments for schizophrenia and involve the views of people with a diagnosis of a mental illness and their carers. Although such views are well known to organisations like the NSF, they have not been formally collected in a research sense in any great number. Of all the studies reviewed recently by Adams and the NHS Centre for Reviews and Disseminations (1999) less than a handful asked individuals for their own views on what worked. It is a subject of endless concern within organisations that work with and represent people with mental health problems that the people in receipt of treatment have not been asked their views. It is clear to us that when people are involved they are more likely to engage fully with a service and achieve a better outcome.

In order to fill this subjective gap the NSF, Mind and the Manic Depression Fellowship surveyed over 2600 people with a mental illness (schizophrenia, manic depression and depression). A summary of the findings opinion \& debate 
from this survey were published in December 2000. A Question of Choice is available from NSF and can be accessed via our website at nsf.org.uk/information /

opinion \& debate research. Nearly two thirds (62\%) of respondents had not been offered a choice of medicine and nearly half $(46 \%)$ had not received written information about sideeffects. The most frequently experienced side-effects were loss of energy (63\%) and weight gain (62\%). Both of these were tolerated by less than $40 \%$ of people.

A consistent finding from the survey was that in the anti-psychotic group atypicals were significantly associated with positive outcomes when compared to typical drugs.

What becomes clear from talking and listening to the views of people with mental illness and their carers is that they want informed choice. They do not want one medicine alone, or one group, they want the widest access possible to find the best fit. It is hoped that the
NICE guidelines will support this pragmatic view and enable all those involved in mental health care to work together to increase people's quality of life.

\section{References}

ADAMS, C. \& NHS CENTRE FOR REVIEWS AND DISSEMINATIONS (1999) Effective Health Care Bulletin, 5, no 6.

HOGMAN, G. (1996) is Cost a Factor? A Survey by the National Schizophrenia Fellowship of the Experiences and Views of Psychiatrists on New Drugs for the Treatment of Schizophrenia. London: NSF.

Gary Hogman Head of Policy and Campaigns, National Schizophrenia Fellowship, 30 Tabernacle Street, London EC2A 4DD
TAYLOR, D. (1999) Is Costa Factor-II? A Survey of Psychiatrists and Health Authorities to Determine the Factors influencing the Prescribing and Funding of Atypical Antipsychotics (eds M. Sharman \& M. Shubhra). London: NSF.

Psychiatric Bulletin (2001), 25, 290-291

\section{DAVID HEALY}

\section{Evidence biased psychiatry? ${ }^{\dagger}$}

For a variety of reasons evidence-based medicine is currently in vogue. The evidence most commonly appealed to comes from randomised controlled trials (RCTs), even though the creator of the RCT, Austin Bradford Hill, argued in the 1960s that while it was good to see some swing toward using RCTs, if we ever ended up thinking that RCTs were the only method to evaluate a treatment the pendulum would not only have swung too far, it would have come off its hook (Hill, 1966).

In common with any other scientific experiment, RCTs are designed to test a null hypothesis. That debriefing after trauma, for example, is no better than non-intervention. These evaluative methods work best when they stop therapeutic bandwagons in their tracks. In the case of the first clinical trials with psychotropic agents, the results were such that sceptics could not readily maintain the position that there was no treatment effect with these drugs, however uncertain they might have remained about the merits of treatment in the longer run.

The majority of recent psychotropic drug trials are business rather than scientific exercises, constructed for the purposes of achieving regulatory approval and thereafter market penetration. In the case of regulatory applications for the newer antipsychotics, adopting the null hypothesis for the data leaves fair-minded observers unable to maintain the position that these drugs are without effect. Some recent studies have included comparator arms, using haloperidol in particular. But none of these studies have led to a regulatory labelling of

$\uparrow$ See editorial, pp. 281-282 and pp. 284-286, pp. $287-288$ pp. $289-290$ and pp. 291-292 the newer agents as superior to or preferable to haloperidol. In the absence of regulatory indications that the null hypothesis has not been shown to hold when new and older agents are compared, it is difficult to see how the makers of guidelines can make many statements comparing agents. It also becomes possible to see why those who might frame guidelines leave themselves open to a legal challenge from pharmaceutical companies, as has happened in other areas of medicine.

There are many statisticians who doubt the power of even well-designed RCTs to generalise to the real world (Gigerenzer, 1993). Company sponsored RCTs invariably recruit samples of convenience, which by definition do not readily sustain an extrapolation to normal clinical practice. In addition, senior investigators on the trials for some of the newer antipsychotics have been jailed, for reasons that leave considerable uncertainty as to how many of the patients in these trials actually existed and how well they were assessed (Stecklow \& Johannes, 1997). Nevertheless, the weight of trial data makes it all but impossible to maintain the position that these new agents are without effect. There can, however, be considerable uncertainty as to how these effects translate into clinical practice. Epidemiologists with doubts about the generalisability of RCT data would prefer harder end-points, such as return to work or suicide figures from large simple trials. Using RCTs, the extrapolation from treatment effects to treatment efficacy would be more convincing if effects were demonstrated across a range of measurement domains from physician-based disease specific scales, through patientbased disease specific and physician-based global functioning scales to patient-based global functioning or quality of life scales. This has not been demonstrated for either new or older agents. Even if it had been demonstrated and the results for shorter-term efficacy were convincing, there could be doubts about the longer-term effectiveness of treatment, owing to the unexplored impact of withdrawal syndromes (Tranter \& Healy, 1998). 